\title{
Light elements in massive single and binary stars
}

\author{
N. Langer ${ }^{1,2}$, I. Brott ${ }^{2}$, M. Cantiello ${ }^{2}$, S. E. de Mink$^{2}$, R. G. Izzard ${ }^{3}$, \\ and S.-C. Yoon ${ }^{1}$ \\ ${ }^{1}$ Argelander-Institut für Astronomie, Universität Bonn, \\ Auf dem Hügel 71, Germany \\ ${ }^{2}$ Sterrenkundig Instituut, University of Utrecht, \\ Postbus 80000, NL-3508TA, Utrecht, the Netherlands \\ ${ }^{3}$ Institut d'Astronomie et d'Astrophysique, Université Libre de Bruxelles, \\ Boulevard du Triomphe, 1050 Brussels
}

\begin{abstract}
We highlight the role of the light elements ( $\mathrm{Li}, \mathrm{Be}, \mathrm{B})$ in the evolution of massive single and binary stars, which is largely restricted to a diagnostic value, and foremost so for the element boron. However, we show that the boron surface abundance in massive early type stars contains key information about their foregoing evolution which is not obtainable otherwise. In particular, it allows to constrain internal mixing processes and potential previous mass transfer event for binary stars (even if the companion has disappeared). It may also help solving the mystery of the slowly rotating nitrogen-rich massive main sequence stars.
\end{abstract}

Keywords. Stars: abundances, binaries

\section{Introduction}

A large effort has been undertaken in the last decades to measure and understand the surface chemical composition of massive main sequence stars. In particular, the detection of nitrogen enhancements in quite a number of such stars (e.g., Gies \& Lambert 1992) has triggered the idea that internal mixing processes can bring material from the stellar core to the surface in rapid rotators (Meynet \& Maeder 2000, Heger \& Langer 2000).

The picture has become more complicated by the recent analysis of a large sample of early B type main sequence stars of Hunter et al. (2008b), who showed that the nitrogenrich stars found by Gies \& Lambert (1992), who restricted their analysis to objects with low projected rotational velocities, are likely part of a population of intrinsically slowly rotation main sequence stars. This view is supported by the work of Morel et al. (2006, 2008), who indeed identifies such a population in our Galaxy (see also Morel 2009). The origin of the nitrogen enrichment in these stars is not understood, but as they are slow rotators it appears difficult to reconcile them with the idea of rotational mixing.

On the other hand, Hunter et al. (2008b) also identified a nitrogen-rich population of rapidly rotating early $\mathrm{B}$ stars, which appears to be well in line with the predictions of theoretical models including rotational mixing (cf., Maeder et al. 2008). The caveat here is that evolutionary models of massive close binaries - whether or not they include rotationally induced chemical mixing (Langer et al. 2008) - appear to predict essentially the same trend of nitrogen enrichment with rotational velocity as the single star models (Meynet \& Maeder 2000, Heger \& Langer 2000).

The light elements lithium, beryllium and boron may play a key role to resolve this issue. They are so rare in the interstellar medium that they can not influence the course of stellar evolution, and thus are often neglected in massive star models. Furthermore, 
they are fragile nuclei which are generally not synthesised in stars, but rather destroyed, at least certainly on the main sequence. In the cool low-mass stars, lithium is most interesting, as it can be observed rather easily, and it is indeed used extensively to constrain internal mixing processes as can be seen in many contributions to this book. In massive stars this role can be played by boron as will be outlined below.

\section{Single stars}

Other than helium and the major CNO nuclei, the light elements $\mathrm{Li}, \mathrm{Be}$, and $\mathrm{B}$ are destroyed by proton capture relatively close to the stellar surface. For both stable boron isotopes, ${ }^{10} \mathrm{~B}$ and ${ }^{11} \mathrm{~B}$, the life time against proton capture is equal to the main sequence life time of a $10 M_{\odot}$ star $\left(10^{7} \mathrm{yr}\right)$ at a temperature of roughly $710^{6} \mathrm{~K}$. Fliegner et al. (1996) have computed the evolution of stars of $15 M_{\odot}$, and found this temperature to occur sufficiently deep inside the stellar envelope (i.e. roughly $1 M_{\odot}$ below the surface; cf. Fig. 1) that its surface abundance can not be altered due to mass loss alone on the main sequence in the B star regime. Thus, the boron abundance in B stars is a critical test of mixing processes in the upper stellar envelope, while CNO and helium abundances additionally trace the mixing in deeper layers.

Models which include rotational mixing show that boron depletion at the stellar surface is predicted for initial rotational velocities above $50 \mathrm{~km} / \mathrm{s}$. However, while in low-mass stars, the rotational velocity is a strong function of age, and so is the lithium abundance, a clear correlation of boron with stellar age is not expected in a population which contains initially fast and slow rotators (cf. Gies \& Lambert 1992). However, a population synthesis simulation by Ines Brott shows (Fig. 2), that a clear correlation of the surface boron depletion with the stellar rotation rate can be expected in early $\mathrm{B}$ type single stars.

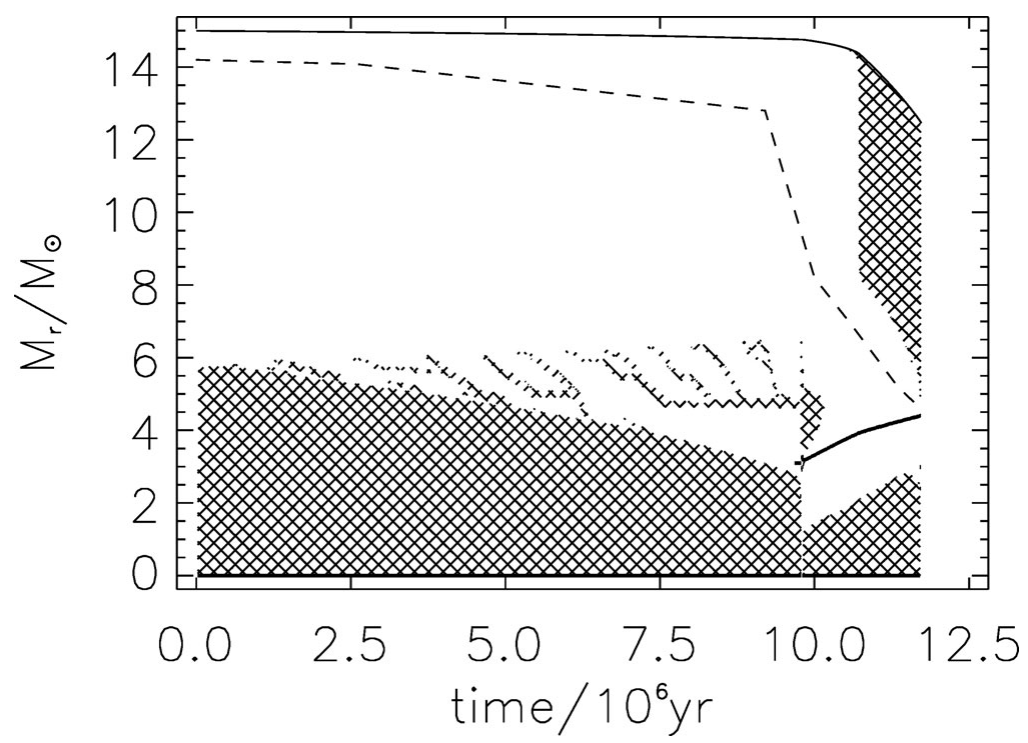

Figure 1. Internal structure of a $15 M_{\odot}$ star during core hydrogen and helium burning. The solid line on top indicates the total mass of the star as function of time. Hatched areas designate convectively unstable mass zones in the star. The full drawn line at $M_{r} \simeq 4 M_{\odot}$ and $t \gtrsim 10^{7} \mathrm{yr}$ designates the location of the H-burning shell during core helium burning. The dashed line indicates the threshold temperature for boron destruction (cf. Fliegner et al. 1996). 
B-stars: B

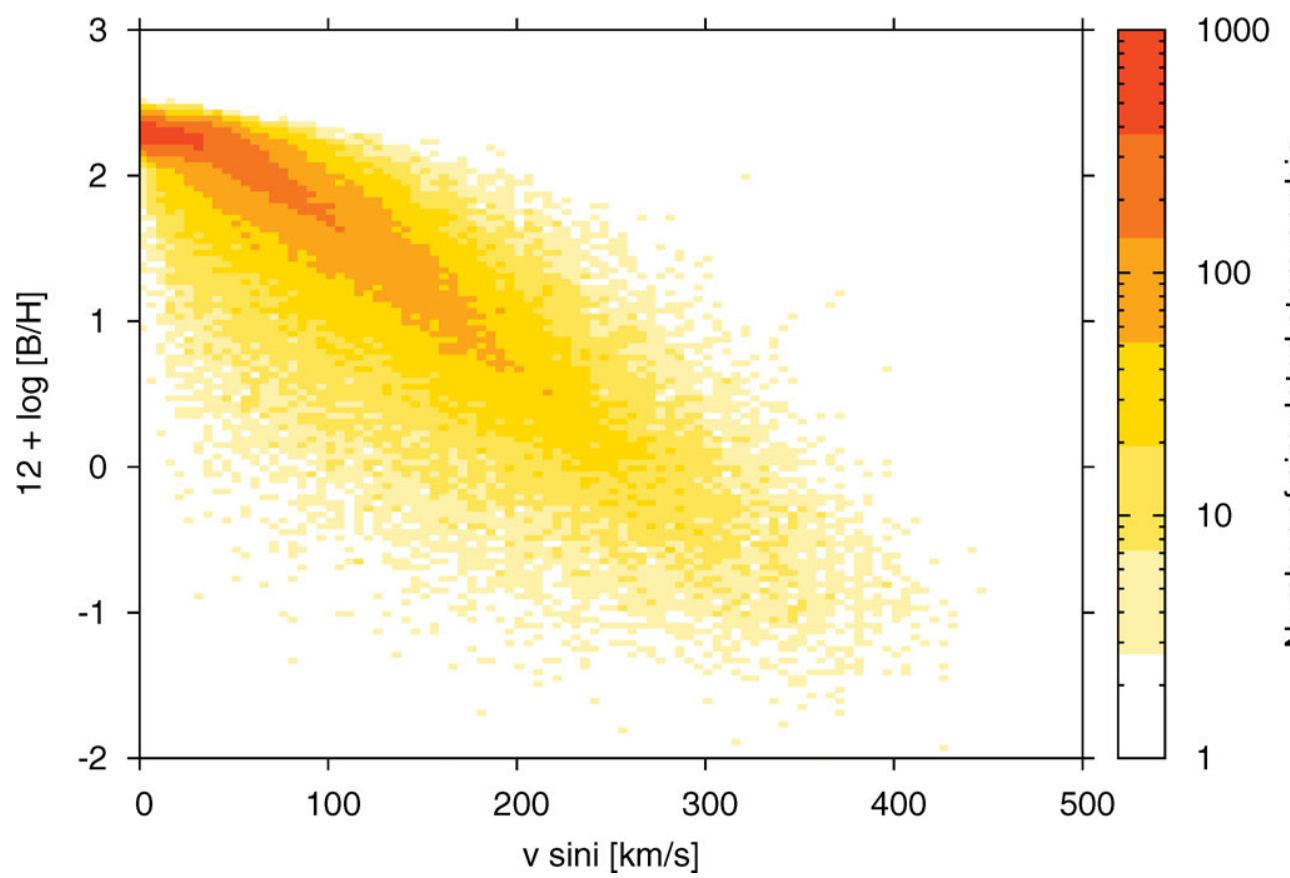

Figure 2. Result of a population synthesis calculation for massive main sequence stars (Brott et al., in prep.), employing a Salpeter initial mass function, and a distribution of initial stellar rotation rates as derived by Hunter et al. (2008a), and a constant star formation rate, based on single star evolution models which include rotational mixing. A random Gaussian error of 0.2 dex was added to the predicted boron abundances.

Figure 3 shows the behaviour of the surface abundances of a rotating $15 M_{\odot}$ in a plot of boron depletion versus nitrogen enhancement. Interestingly, it predicts that boron depletion happens essentially before nitrogen enrichment occurs. Thus, stars which are already depleted in boron, but which are still nitrogen normal are expected. Indeed, in the sample of Venn et al. (1996), several such stars seem to exist. The importance of this finding becomes more clear in the next section.

\section{Binary stars}

The binary fraction of massive stars is very high, and it may be futile to try to understand their surface abundances without considering effects of binarity (Langer et al. 2008). The issue of binarity is not easily resolved, because of two reasons.

Firstly, after a strong binary interaction, the object may not appear to be a binary any more. This is so since many mass transfer systems will produce a rejuvenated main sequence star which dominates the light of the system, with a faint helium star in a wide orbit. In fact, many such objects ought to exist, as we see many of their descendants, the Be-X-ray binaries. However, for the stage where the main sequence star has a helium star companion, we practically do not know any counterpart. Also, there will be many cases where the post-interaction system is in fact a single star. E.g., in many systems consisting of a main sequence star and a helium star companion, the supernova explosion of the helium star will disrupt the binary (instead of leading to a Be-X-ray binary stage). And furthermore, as many as $10 \%$ of all massive stars may actually merge with their close 


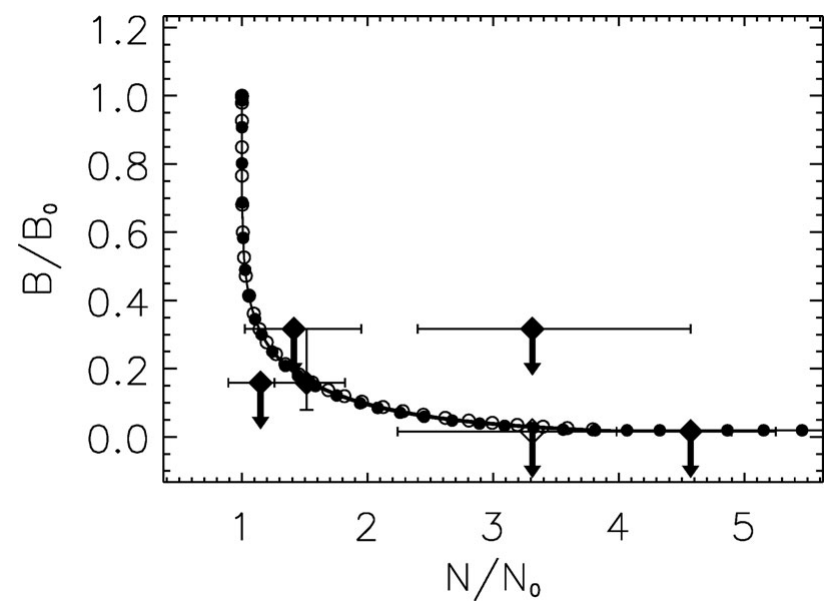

Figure 3. Boron depletion versus nitrogen enrichment for rotating stars of $10 M_{\odot}$ during the main sequence evolution (Fliegner et al. 1996). Open circles correspond to a moderately, filled circles to a fast rotating model. Two neighbouring symbols on each line indicate a constant distance in time of $10^{6} \mathrm{yr}$. Diamonds indicate the location of the five B main sequence stars (filled symbols) and one B giant (open symbol) of Venn et al.'s (1996) sample (see also Venn et al. 2002). Arrows designate upper limits.

companion during the main sequence evolution. Therefore, most of the binaries which we detect in massive main sequence star populations may have in fact not yet interacted, while on the other hand, many apparent single stars may be the result of a strong binary interaction.

Secondly, binary interaction has many complex branches, and it is a big theoretical enterprise to even fully consider the most important ones in a population study. So far, no such study exists which takes the physics of rotation fully into account - which is what appears to be needed in order to demonstrate that rotational mixing works in Nature.

However, there are some binary evolution models available which include all the required physics, and while they will not allow to obtain a full view of the picture, they may give indications in one or the other direction. To that purpose, Fig. 4 shows the time evolution of the surface rotational velocity and of the surface boron abundance of the mass gainer (i.e. the star which will be visible after the mass transfer) of a close massive binary. The lower most panel also shows the boron depletion factor as function of the rotational velocity in this star.

The model shown in Fig. 4 does include rotational mixing, which produces the mild boron depletion before the first mass transfer event (at about $t=8.5 \mathrm{Myr}$ ). The mass transfer event itself, which occurs on a time scale of some $10^{4} \mathrm{yr}$, puts boron depleted layers on the surface of the mass gainer, and subsequent thermohaline mixing brings the surface boron abundance back to a level of one per mille of the initial boron abundance.

Consecutively, the mass gainer is spun down by tidal interaction, while rotational mixing reduces the surface boron abundance slightly more. As a result of this phase, one obvious result from Fig. 4 is that in mass gainers of close binaries, one can not generally expect a correlation of the boron depletion factor with the surface rotation rate. Here, a slowly rotating main sequence star is produced which shows a surface boron depletion by 3 to 4 orders of magnitude. Fig. 2 shows that such a strong depletion is only expected in single stars with rotation rates above $300 \mathrm{~km} / \mathrm{s}$.

The model in Fig. 4 suffers from a second mass transfer at $t \simeq 10.2 \mathrm{Myr}$, which leads to a second spin-up of the mass gainer and a further strong reduction of the surface boron 


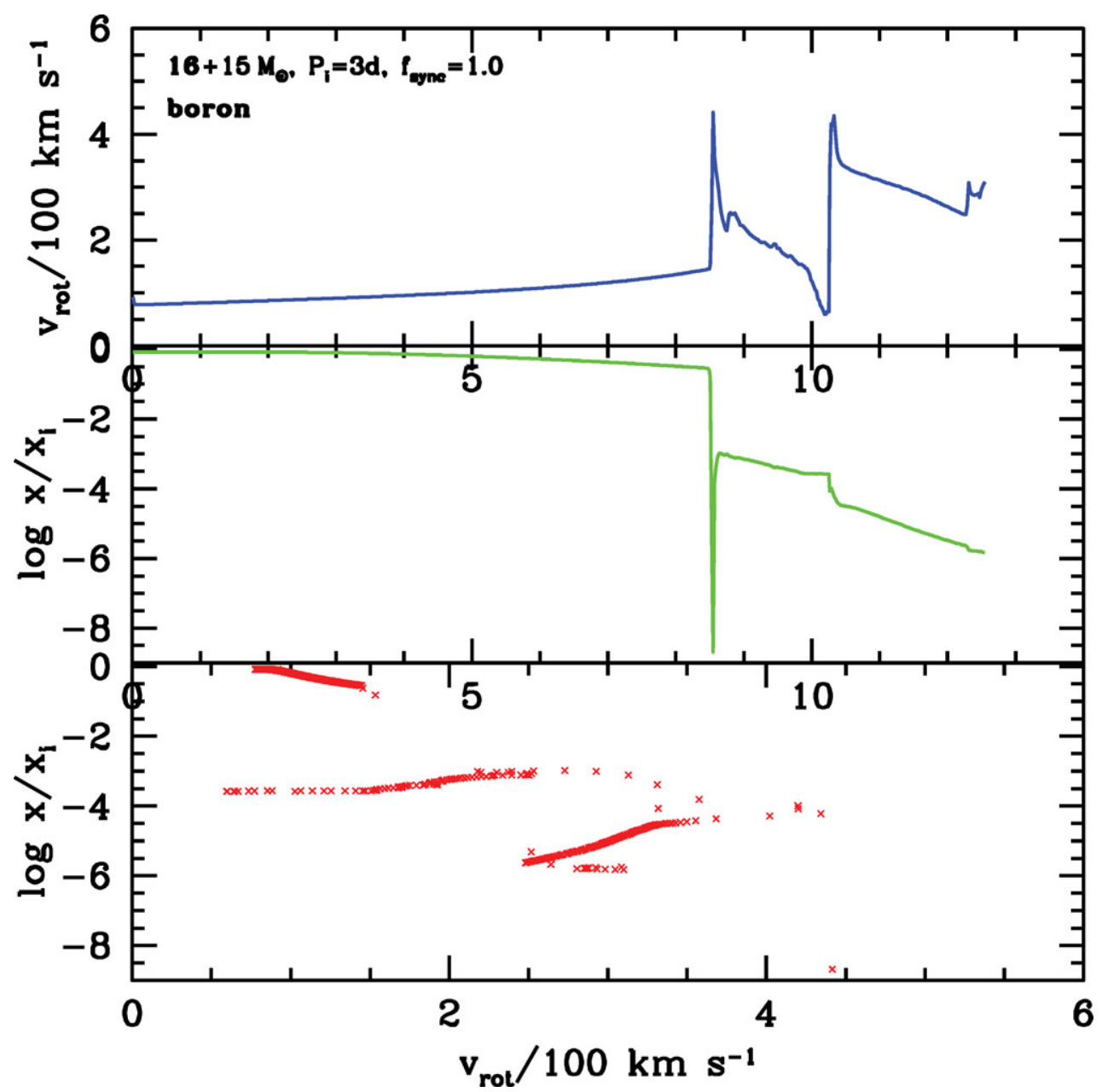

Figure 4. Equatorial rotational velocity (upper panel) and surface boron mass fraction relative to the initial value (middle panel) as function of time (in Myr), for the mass gainer in a solar metallicity $16 M_{\odot}+15 M_{\odot}$ binary with an initial orbital period of 3 days. The computations include the physics of rotation for both components as in Heger et al. (2000), and Spin-Orbit coupling as in Detmers et al. (2008) with the nominal coupling parameter $f_{\mathrm{sync}}=1$, and rotationally enhanced stellar wind mass loss (Langer 1998). Internal magnetic fields are not included. The bottom panel shows the evolution of the mass gainer in the boron depletion versus rotational velocity diagram, where each data point represents a duration of $20000 \mathrm{yr}$. The spin-down of the star after the first accretion event $(t=8.5 \ldots 10 \mathrm{Myr})$ is mostly due to tidal effects.

abundance. As the orbit widens strongly during the second mass transfer phase, no tidal spin-down occurs thereafter. The mass gainer is now a rapidly rotating main sequence star which is strongly boron depleted.

While rotational mixing is included in this model, we want to point out that the effects of rotational mixing and of mass transfer (and thermohaline mixing) are well separable in Fig. 4. The steps in the time evolution of the boron abundance are produced by the mass transfer, and the long time scale changes are due to rotational mixing. We conclude that the main features of the boron evolution of this model would not change if rotational mixing were switched off. From this consideration, we can argue that if rotational mixing would not operate in Nature, then perhaps binary models would not 


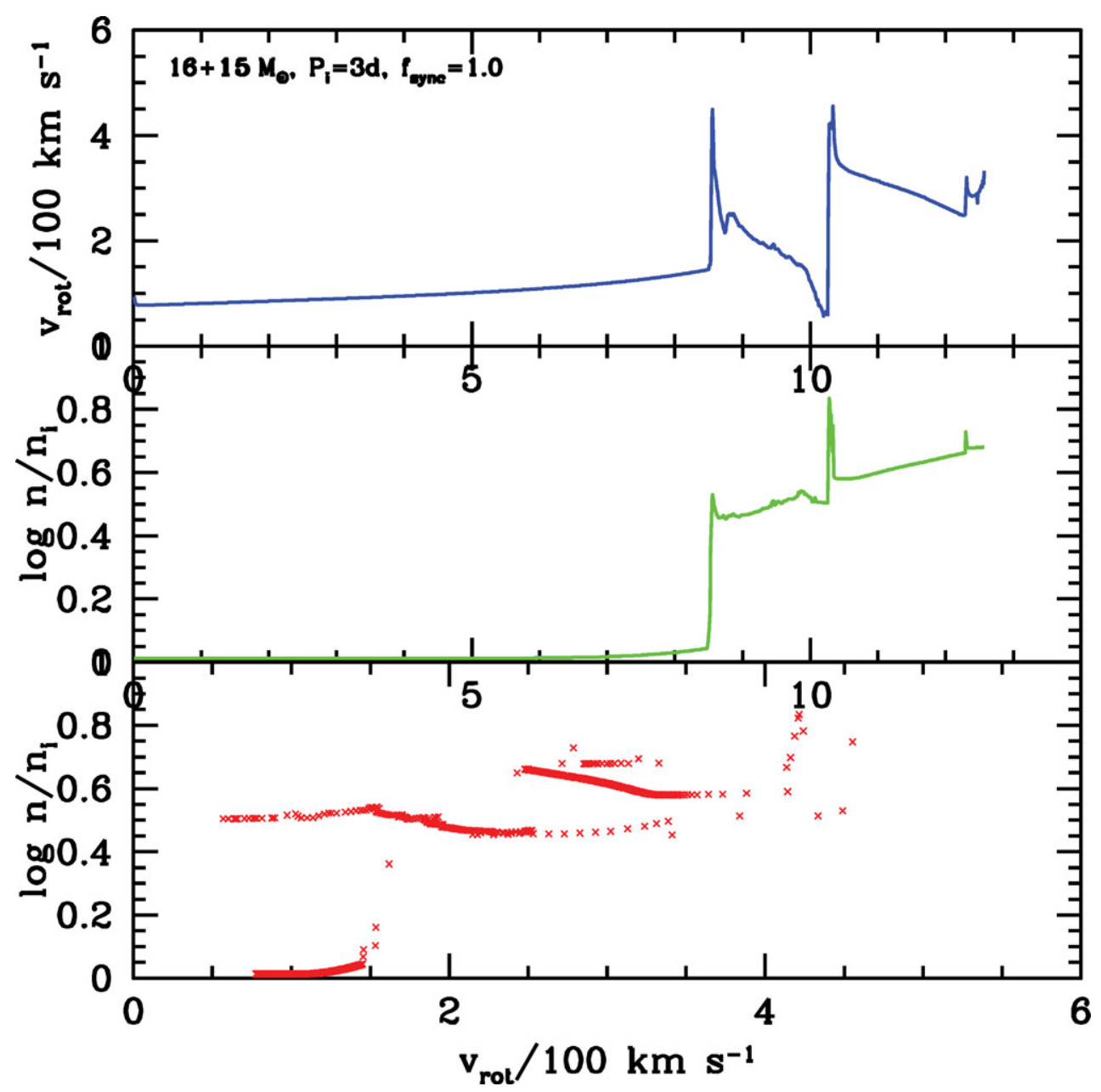

Figure 5. As Fig. 4, but showing the surface nitrogen abundance.

predict main sequence stars with only a mild boron depletion (unless one considered masses much higher than $15 M_{\odot}$, where mass loss can gradually uncover boron-depleted layers).

The situation becomes more clear when nitrogen is considered at the same time. Fig. 5 shows the nitrogen surface abundances of the same binary model described above. The nitrogen surface abundance increases abruptly due to the mass transfer. Thus, from this sequence, one would not expect to observe stars which are boron depleted but not nitrogen enriched unless rotational mixing operates. The corresponding stars in Fig. 2 thus indicate that rotational mixing is perhaps operating as we expect.

However, this is not yet a definite conclusion. The example binary displayed in Figs. 4 and 5 evolves rather conservatively, i.e. about $70 \%$ of the transferred matter is actually accepted by the mass gainer (while the rest is ejected due to its excess angular momentum). We know from observations and predict theoretically that many mass transfer systems evolve rather non-conservatively (Petrovic et al. 2005ab). We can currently not exclude that some of these systems produce boron depletion with only mild or no nitrogen enhancement. 

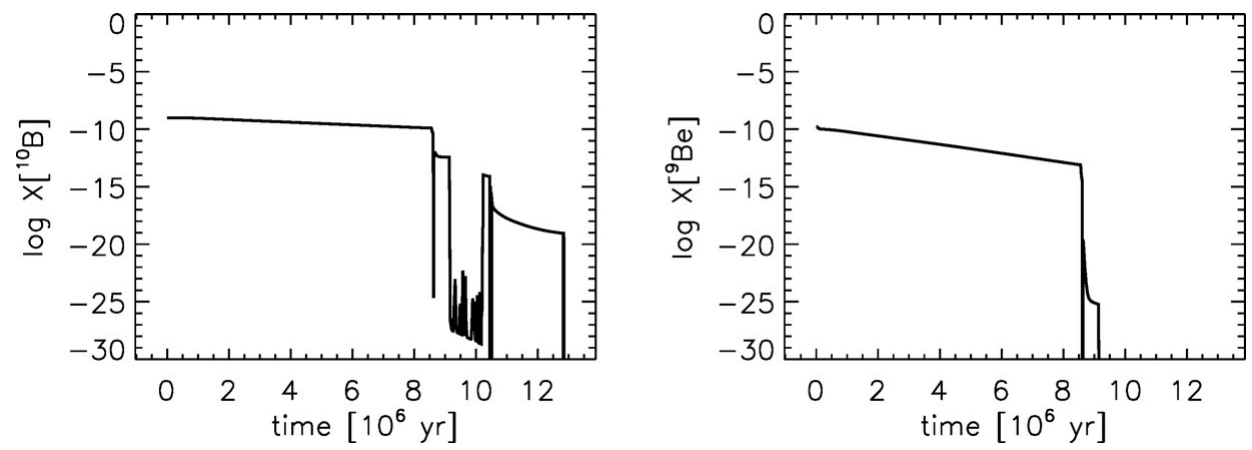

Figure 6. Surface abundance of boron 10 (left) and of beryllium (right) as function of time during core hydrogen burning, for the mass gainer in a solar metallicity $16 M_{\odot}+15 M_{\odot}$ binary with an initial period of 3 days. In contrast to the models shown in Figs. 4 and 5, the stellar models shown here have been computed including angular momentum transport by internal magnetic fields (Yoon et al., in prep.).

It is also interesting to consider boron as a test of rotational mixing in very close and very massive pre-mass transfer binaries, as explored by de Mink et al. (2009). In such very tight binaries, tidal synchronisation can enforce very rapid rotation of both stars. While de Mink et al. point out that generally the nitrogen surface abundance is the prime observable for such test, their results on boron depletion appear particularly interesting for Galactic binaries, since in those boron has a larger predicted relative change then nitrogen.

Finally, in Fig. 6, we show the surface abundances of boron and of beryllium in a similar binary as the one discussed above (even though some physics assumptions were different, which is not essential for our discussion here). Fig. 6 indicates that also beryllium is very interesting from the theoretical point of view. However, beryllium abundance determinations in hot main sequence stars appear to be difficult.

\section{Unknown mixing processes}

We have seen in the previous sections, that the surface abundances of massive main sequence stars are not yet fully understood, and that unambiguous evidence for the existence of rotationally induced mixing in massive stars is still lacking. Of particular worry is the solid evidence for a population of nitrogen-rich slowly rotating massive main sequence stars (Hunter et al. 2008b, 2009; Morel et al. 2006, 2008; Morel 2009). While the binary models discussed above do show a way to produce such stars (Langer et al., 2008; cf. Fig. 5), the Galactic fraction of this population contains well investigated $\beta$ Cephei pulsators (Morel et al. 2006, 2008) none of which seems to show any indication of binarity. Despite the warning above that binarity might be difficult to detect in postmass transfer systems, the lack of any indication of a companion in well-studied nearby stars could imply that binarity is not the (only) answer to this question. It also remains to be seen whether binary evolution could produce enough of these objects, which may have a frequency of about $15 \%$ of all main sequence stars (Hunter et al. 2008b).

So we may face the situation that so far completely unaccounted mixing processes may operate in stars (see also Brott et al., in preparation). Perhaps, they could be related to magnetic fields in the interior of massive stars. Also gravity waves could be excited in massive stars, as Talon \& Charbonnel (2008) employ them for angular momentum transport in intermediate-mass stars. However, we want to end with a related possibility, for which recent observational evidence has accumulated. 


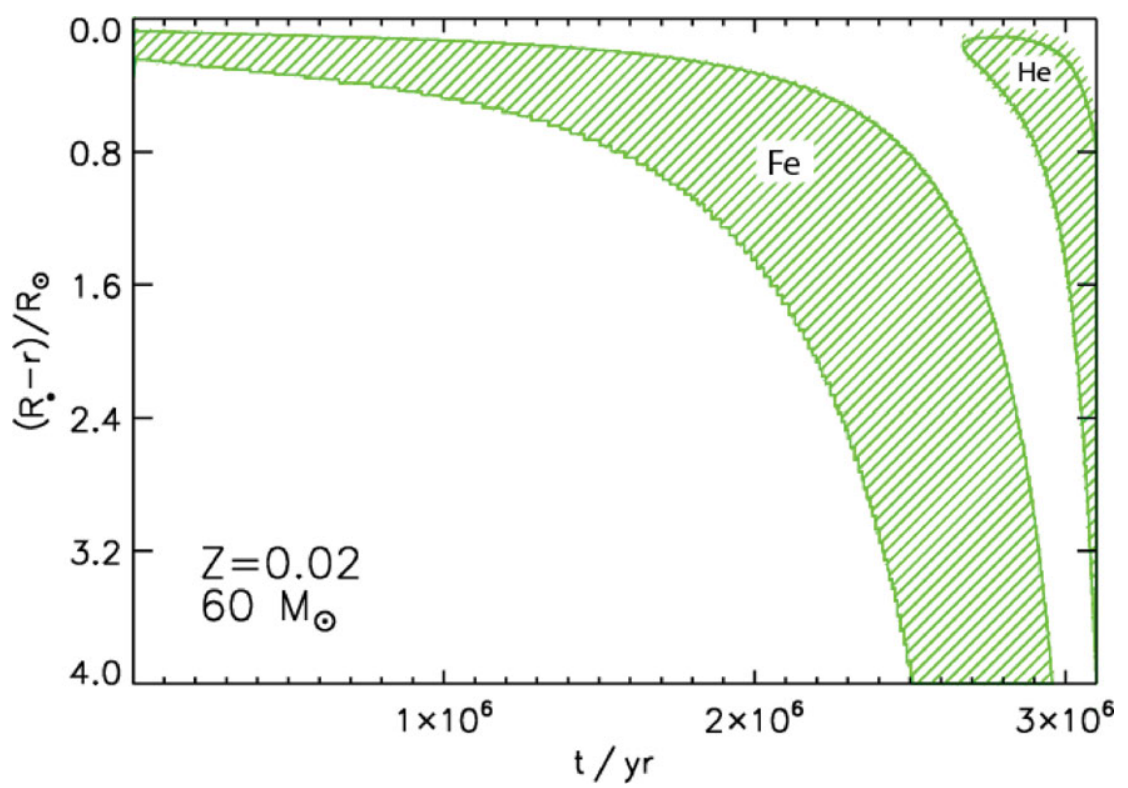

Figure 7. Evolution of the radial extent of the subsurface helium and iron convective regions (hatched) as function of time, from the zero age main sequence to roughly the end of core hydrogen burning, for a $60 M_{\odot}$ star (Cantiello et al. 2009). The top of the plot represents the stellar surface. Only the upper $4 R_{\odot}$ of the star are shown in the plot, while the stellar radius itself increases during the evolution. The star has a metallicity of $\mathrm{Z}=0.02$, and its effective temperature decreases from $48000 \mathrm{~K}$ to $18000 \mathrm{~K}$ during the main sequence phase.

Cantiello et al. (2009) investigated the subsurface convection zones which occur in the envelopes of hot massive stars due to the iron opacity peak (cf. Fig. 7). They found that the occurrence of strong subsurface convective motion predicted by the models correlates with observed large microturbulent velocities deduced from stellar spectroscopy. While this may not be sufficient to conclude that subsurface convection causes observable motion at the stellar surface, it appears to be a possibility. This could mean that the subsurface layers of massive stars, independent of their rotation, could be in motion, and perhaps lead to some mixing near the surface; this might produce a surface boron depletion without changing nitrogen.

\section{Conclusions}

It remains a major challenge to the theory of massive star evolution to explain the observed surface abundances of massive main sequence stars. While until recently, the incorporation of rotational mixing was thought to lead to a much better agreement, the discovery that a significant fraction of early B dwarfs are nitrogen-rich and intrinsically slowly rotating has cast some doubts on the previous ideas. We argue that boron observations of early type main sequence stars, as performed by Venn et al. $(1996,2002)$ and Morel et al. (2006, 2008), have the potential to move towards a solution. Clearly, binary evolution needs to be considered at the same time. Finally, we may be facing the situation that still not all mixing processes which can operate in massive main sequence stars have been described. Amongst possible candidates is mixing due to magnetic processes, and mixing induced by subsurface convection zones in hot massive stars. 


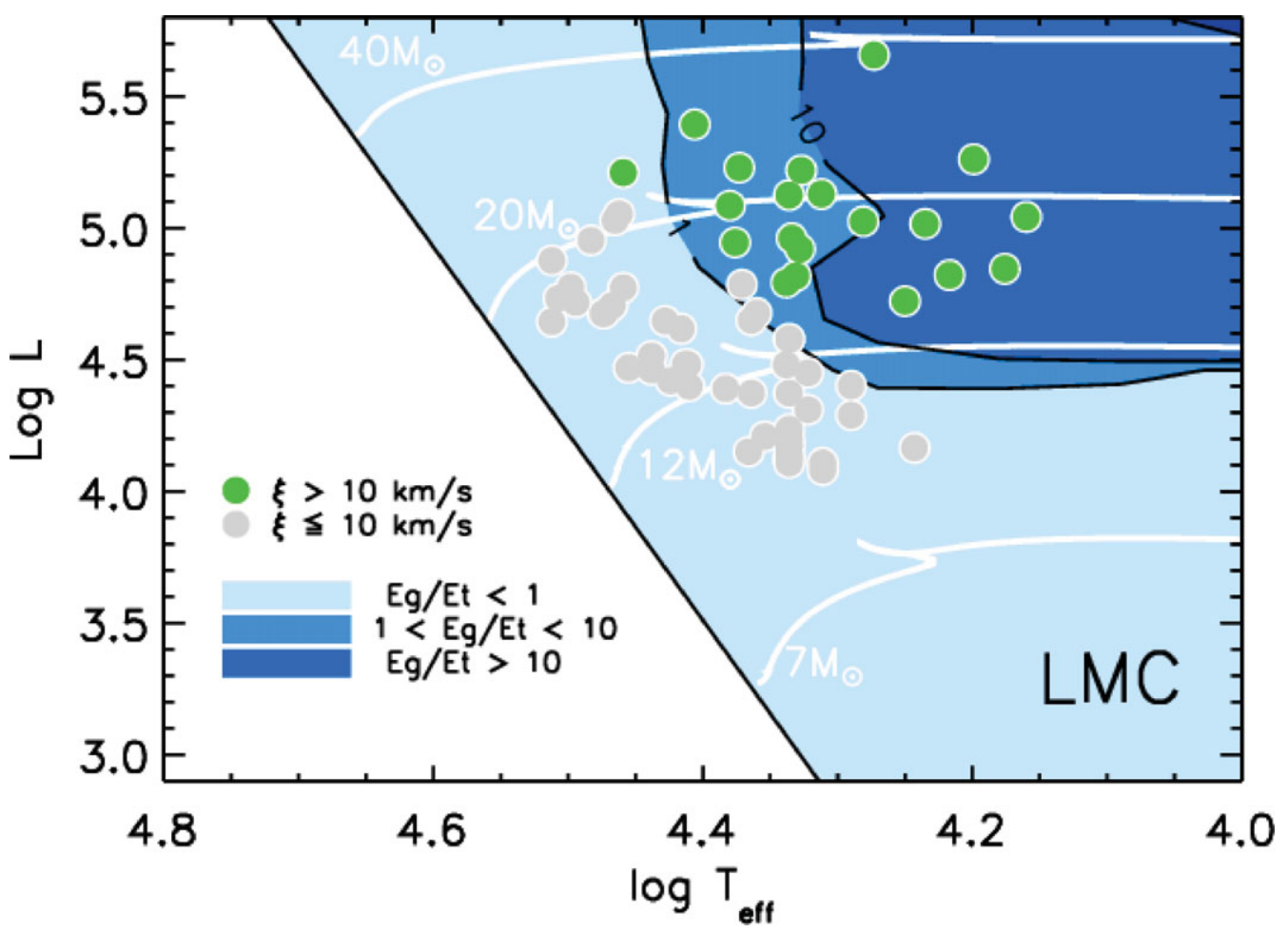

Figure 8. Values of the ratio $E_{\mathrm{g}} / E_{\mathrm{s}}$ of the kinetic energy in the form of gravity waves above the iron convection zone, to the kinetic energy of the surface velocity field, as a function of the location in the HR diagram (Cantiello et al. 2009). This plot is based on evolutionary models between $5 \quad M_{\odot}$ and $100 \quad M_{\odot}$ for LMC metallicity. The ratio $E_{\mathrm{g}} / E_{\mathrm{s}}$ (see Eq. (9) of Cantiello et al. 2009) is estimated using a value $v_{\mathrm{s}}=10 \mathrm{~km} \mathrm{~s}^{-1}$ for the surface velocity amplitude. Overplotted as filled circles are stars which have photospheric microturbulent velocities $\xi$ derived in a consistent way by Hunter et al. (2008a). Only data for stars with an apparent rotational velocity of $v \sin i<80 \mathrm{~km} \mathrm{~s}^{-1}$ is plotted. Solid white lines are reference evolutionary tracks, and the full drawn black line corresponds to the zero age main sequence.

\section{References}

Cantiello, M., Langer, N., Brott, I., de Koter, A., Shore, S. N., Vink, J. S., Voegler, A., Lennon, D. J., \& Yoon, S.-C. 2009, A\& A, 499, 279

de Mink, S. E., Cantiello, M., Langer, N., Pols, O. R., Brott, I., \& Yoon, S.-Ch. 2009, A\&A, 497, 243

Detmers, R. G., Langer, N., Podsiadlowski, Ph., \& Izzard, R. G. 2008, A\&SA, 484, 831

Fliegner, J., Langer, N., \& Venn, K. A. 1996, A\&A (Letters), 308, 13

Gies D. R. \& Lambert D.L 1992, ApJ, 387, 673

Heger, A. \& Langer, N. 2000, ApJ, 544, 1016

Heger, A., Langer, N., \& Woosley, S. E. 2000, ApJ, 528, 368

Hunter, I., Brott, I., Lennon, D. J., Langer, N., Dufton, P. L., Trundle, C., Smartt, S. J., de Koter, A., Evans, C. J., \& Ryans, R. S. I. 2008b, ApJ (Letters), 676, 29

Hunter, I., Lennon, D. J., Dufton, P. L., Trundle, C., Simon-Diaz, S., Smartt, S. J., Ryans, R. S. I., \& Evans, C. J. 2008a, A\& $A, 479,541$

Hunter, I., Brott, I., Langer, N., Lennon, D. J., Dufton, P. L., Howarth, I. D., Ryans, R. S. I.,

Trundle, C., Evans, C. J., de Koter, A., \& Smartt, S. J. 2009, A\&AA, 496, 841

Langer, N. 1998, A\&A, 329, 551

Langer, N., Cantiello, M., Yoon, S.-C., Hunter, I., Brott, I., Lennon, D., de Mink, S., \& Verheijdt, M. 2008, IAU Symposium 250, p. 167 
Maeder, A., Meynet, G., Ekström, S., \& Georgy, C. 2009, Communications in Asteroseismology, 158,72

Meynet, G. \& Maeder, A. 2000, A\&A, 361, 101

Morel, T. 2009, Communications in Asteroseismology, 158, 122

Morel, T., Butler, K., Aerts, C., Neiner, C., \& Briquet, M. 2006, A\&A, 457, 651

Morel, T., Hubrig, S., \& Briquet, M. 2008, A\& A, 481, 453

Petrovic, J., Langer, N., Yoon, S.-C., \& Heger, A. 2005a, A\&A, 435, 247

Petrovic, J., Langer, N., \& van der Hucht, K. A. 2005b, A\&A, 435, 1013

Talon, S. \& Charbonnel, C. 2008, A\&SA, 482, 597

Venn, K. A., Lambert, D. L., \& Lemke, M. 1996, A\&A, 307, 849

Venn, K. A., Brooks, A. M., Lambert, David L., Lemke, M., Langer, N., Lennon, D. J., \& Keenan, F. P. 2002, ApJ, 565, 571 